\title{
Simultaneous Satellite and Ground Based Observations of Polar Cap Phenomena
}

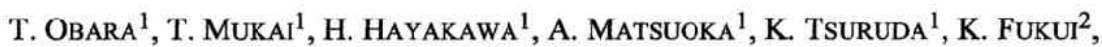 \\ J. V. RodrigueZ ${ }^{2}$, and C. E. VALladares ${ }^{3}$ \\ ${ }^{1}$ Inst. of Space \& Astronautical Sci., 3-1-1 Yoshinodai, Sagamihara, Kanagawa 229, Japan \\ ${ }^{2}$ Phillips Laboratory, Geophysics Directorate, Hanscom AFB, MA 01731, U.S.A. \\ ${ }^{3}$ Inst. of Space Res., Boston College, Newton, MA 02159, U.S.A.
}

(Received September 29, 1995; Revised February 8, 1996; Accepted March 6, 1996)

\begin{abstract}
In order to understand the processes of formation and motion of sun-aligned polar cap arcs as well as patches, we have analyzed simultaneous observations from the Akebono (EXOS-D) satellite and ground based instruments at Qaanaaq. Results demonstrated the correspondence between the localized electron precipitation signatures observed from Akebono and multiple sun-aligned arcs observed using an all sky camera (ASC) at Qaanaaq and have confirmed that sun-aligned arcs correspond to $\operatorname{div} E<0$. We also confirmed the time delay of appearance and disappearance of sun-aligned arcs in response to IMF change. As for patches, most of the patches drifted anti-sunward, but some ceased to drift within field of view of the all sky camera. Results of comparison between patch movement and the convection demonstrated a general consistency between them.
\end{abstract}

\section{Introduction}

All sky imaging and ionospheric sounding have revealed that the polar cap $F$-region is unexpectedly active for both $B z<0$ and $B z>0$ of the interplanetary magnetic field (IMF). Buchau et al. (1983) demonstrated that during quiet periods sun-aligned arcs (SAs) are predominant. SAs disappear during magnetically active periods, and large patches of enhanced ionization drift in the anti-sunward direction. SAs and ionization patches are, hence, the predominant features in the polar cap ionosphere.

Many case studies and statistical studies have been carried out on the sun-aligned arcs (SAs), but the following issues still remain unanswered:

(1) How do SAs appear or disappear in response to IMF polarity change?

(2) Is the arc electrodynamics coherent over its length and readily mapped up to the high-altitude region from the ionosphere?

(3) What is the structure of the magnetosphere which leads to SAs?

(4) Where is the possible source region of the SA's electrons?

By using the simultaneous observation data from the Phillips Laboratory all sky camera (ASC) at Qaanaaq in Greenland together with Akebono observations, we have conducted a systematic analysis with some specific interests; i.e.

(a) to clarify the electrodynamics and its coherency along the arc, and

(b) to investigate the growth and decay of SAs in response to IMF.

When the IMF is directed southward, $F$-region density patches appear in the polar cap region (Weber et al., 1984). There are some questions that need to be addressed;

(1) identification of the patch source and generation mechanism, and

(2) role of the convection in moving and changing the shape of patches.

By using the simultaneous observation data from the Qaanaaq all sky camera (ASC) and Akebono, we have investigated the relationship between the patch movement and the polar cap convection. 


\section{Data Description}

The Akebono (EXOS-D) satellite which was launched on Feb. 22, 1989 has an inclination of 75 deg., and passes over Qaanaaq $\left(77.5^{\circ} \mathrm{N}, 69.2^{\circ} \mathrm{W}\right)$ once per day. We have surveyed the simultaneous observation periods and found 38 passes during the winter months of 1989, 1991 and 1992 . The data for 1990 is not used since the perigee of the Akebono $(\sim 270 \mathrm{~km})$ was just above the northern polar cap region and we could not conduct real time observations using Esrange (Sweden) and Prince Albert (Canada) satellite stations (Obara et al., 1990).

Since the ASC at Qaanaaq has a very high sensitivity, it always observed patches or arcs. The number of events is as follows;

\section{Sun-aligned arcs, Patches, SAs and Patches 16 passes, $\quad 19$ passes, 3 passes.}

The number of the cases when patches and arcs were observed together in the Qaanaaq field of view is small, and the co-existence appeared to be transient. Specifically, SAs were replaced by patches in two passes, vise versa in another pass.

\section{Sun-Aligned Arcs}

\subsection{Identification of localized electron precipitation}

On Akebono satellite, the LEP instrument has been installed which makes comprehensive observations of energy and pitch angle distributions of electrons and ions in an energy range from $10 \mathrm{eV}$ up to $16 \mathrm{keV}$ for both species (Mukai et al., 1990). The LEP instrument observed localized signatures of electron

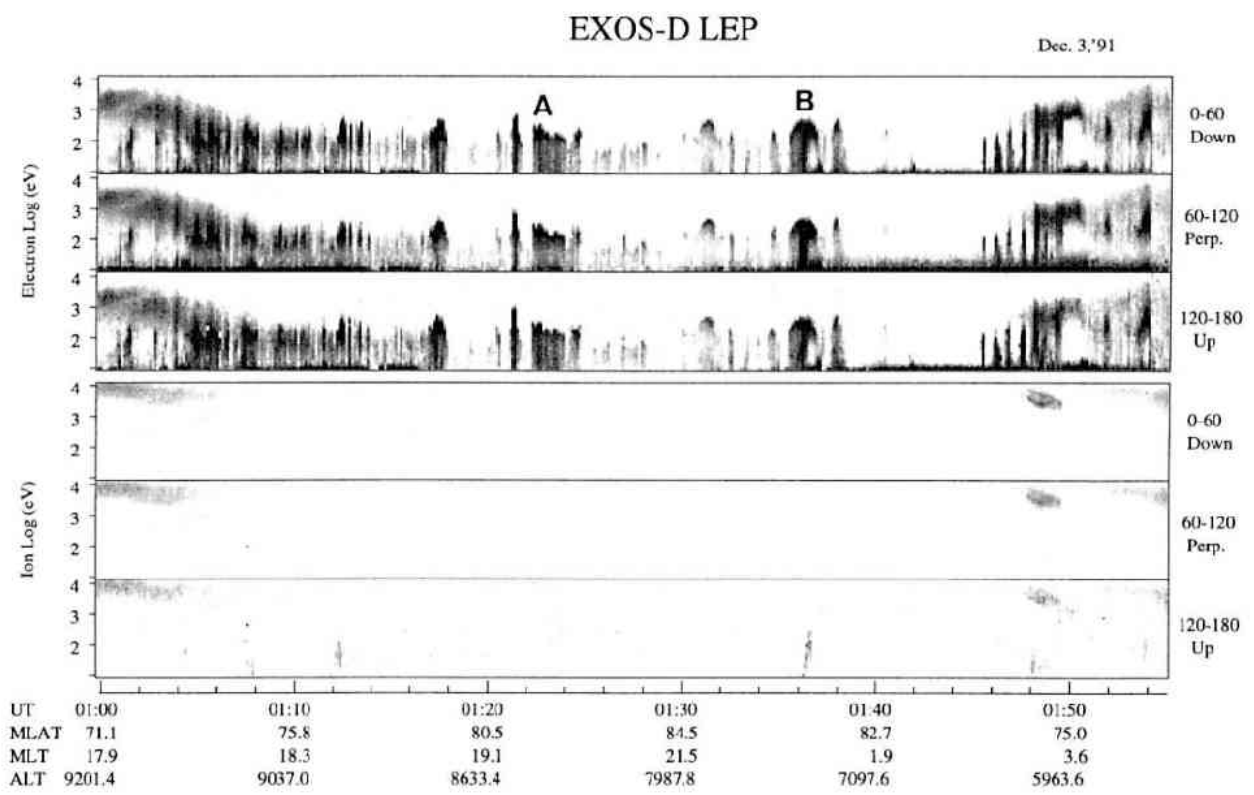

Fig. 1. Akebono E-t diagrams for electrons (top three) and ions (bottom three), sorted by the pitch angles. Energy range is logarithmically scaled in the vertical axis, ranging from $10 \mathrm{eV}$ to $16 \mathrm{keV}$ for both species. The Akebono satellite entered the polar cap region at $01: 18 \mathrm{UT}$ and stayed till $01: 47 \mathrm{UT}$, observing intense electron precipitations at 01:23UT and 01:36UT. 


\section{1/12/03 01:28:46UT}

(a)

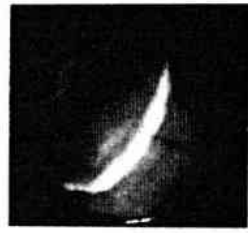

(b)

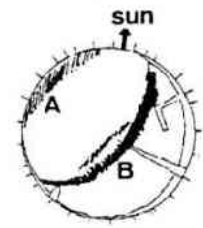

(c)

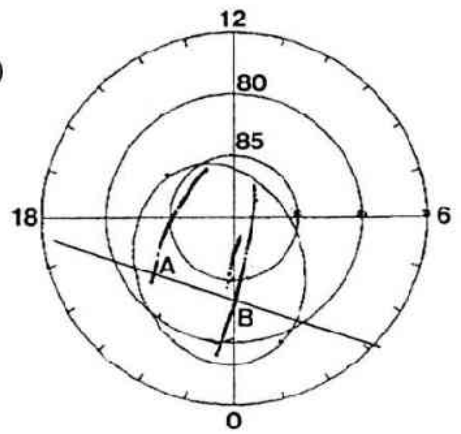

Fig. 2. (a) All sky image taken from Qaanaaq at 01:28:48UT on Dec. 3, 1991 and(b) its sketch. A small arrow directs the sun. (c) The projection of SAs into the polar map.

precipitation which corresponded to the multiple sun-aligned arcs, as expected. Figure 1 demonstrates en example of the Akebono encounter with intense localized electron precipitations in the polar cap region at 01:23UT and 01:36UT on Dec. 3,1991. The 630.0 nm all sky image seen from Qaanaaq at 01:28:46UT and its sketch are given in Figs. 2(a) and 2(b), respectively. The projection of the SAs into corrected geomagnetic (CG) coordinate is given in Fig. 2(c), where we can see two elongated features of sun-aligned arcs were in the polar cap region. We have added the Akebono trajectory on the polar map and marked the exact locations of Akebono's encounter with arcs by "A" and "B", corresponding to the localized electron precipitations marked "A" and "B" in Fig. 1.

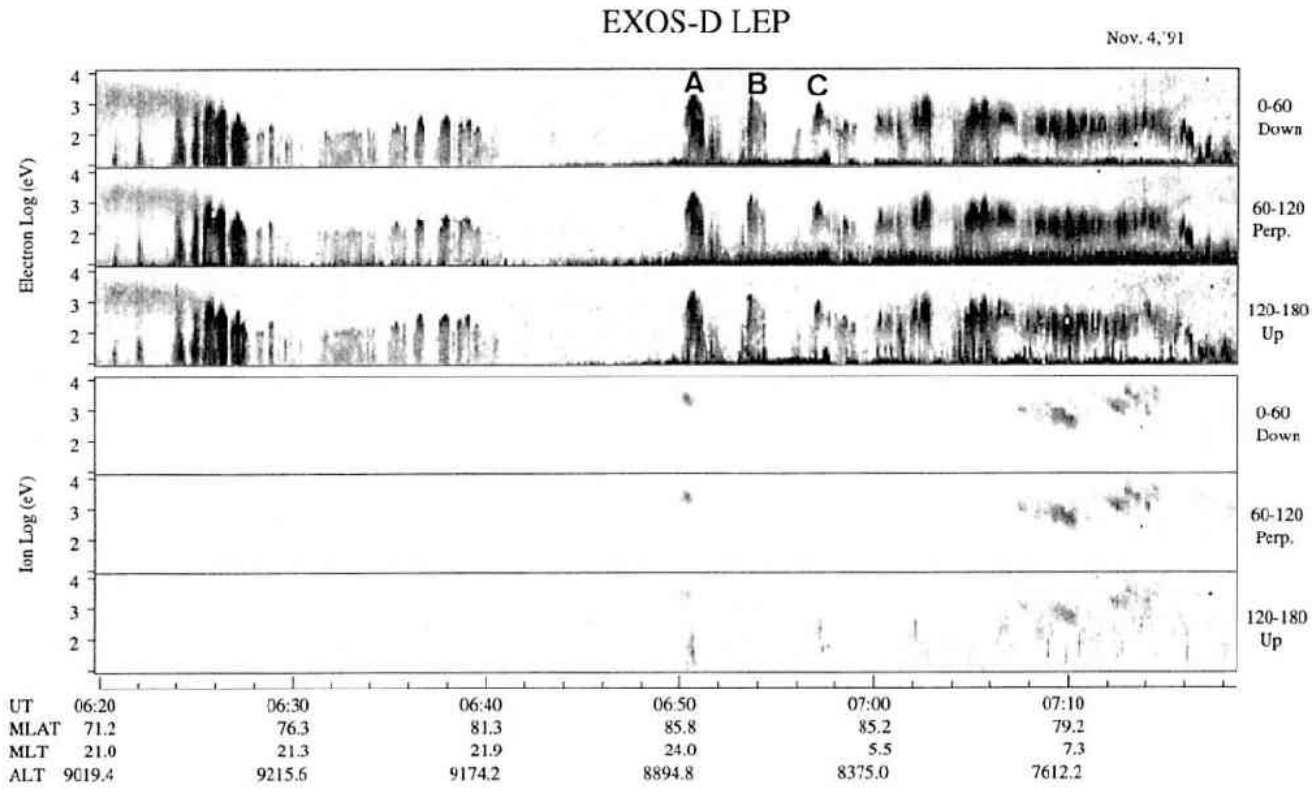

Fig. 3. Same format as in Fig. 1. Observation was made on Nov. 4, 1991. 
(a)

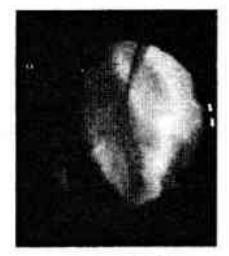

(b)

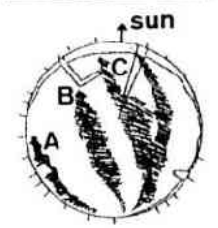

(c)

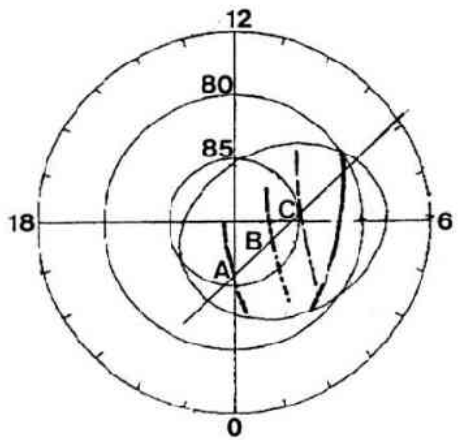

Fig. 4. Same format as in Fig. 2. Observation was made on Nov. 4, 1991.

On Nov. 4, 1991. Akebono encountered three localized electron precipitations at 06:51UT, 06:54UT and 06:57UT as marked "A", "B" and "C" in Fig. 3. Akebono actually observed additional electron precipitations after 07:00UT, but they seem to be in the auroral oval region expanded into very high latitude due to a quiet magnetic condition. The $630.0 \mathrm{~nm}$ all-sky image from Qaanaaq at 06:59:36UT and its sketch are given in Figs. 4(a) and 4(b), respectively. The projection of these SAs together with oval is given in Fig. 4(c), in which the exact locations of Akebono's encounter with arcs are marked by "A", "B" and "C", corresponding to the localized electron precipitations marked "A", "B" and "C" in Fig. 3.

\subsection{Relation of electric field and electron precipitation}

On Akebono satellite, the EFD instrument has been installed which provides quasi-static vector electric field along the orbit (Hayakawa et al., 1990). By using the EFD data we have confirmed an association of negative divergence of the horizontal electric field with a sun-aligned arc. We will first demonstrate Nov. 4, 1991 data for example. The top two panels in Fig. 5 show the sunward $(X)$ and duskward ( $Y$ ) components of the electric field in GSM frame. The satellite traversed the polar cap region from the premidnight $(M L T=21 \mathrm{~h})$ to the morning $(M L T=7 \mathrm{~h})$, passing by the north magnetic pole. Since the satellite moved from the duskside to the dawnside, the positive slope of the $Y$ component gives negative contribution to $\operatorname{div} E$. For the $X$ component of the electric field, the negative slope contributes to $\operatorname{div} E<0$, since the satellite moved from nightside to dayside. The bottom panel in Fig. 5 demonstrates the energy flux of precipitating electrons, where Akebono encountered three SAs at 06:51UT, 06:54UT and 06:57UT. These correspond to SAs labeled "A", "B" and "C" in Fig. 3. It is clear that localized electron precipitation signatures coincide with the $\operatorname{div} E<0$ regions. It is noted that the variation of the $E x$ (sunward component of the electric field) is smaller than that of $E y$ (duskward component of the electric field). This means that the arc is almost sun-aligned and this is consistent to the arc elongation as shown in Fig. 4(c).

Another example is given in Fig. 6. The observation was made on Jan. 15, 1990. In this case, the satellite traversed the polar cap region from the postnoon $(M L T=14 \mathrm{~h})$ to the midnight $(M L T=0 \mathrm{~h})$. Since the satellite moved from dusk to dawn, the positive slope of of the $Y$ component contributes to $\operatorname{div} E<0$. For the $X$ component of the electric field, the positive slope contributes to $\operatorname{div} E<0$, since the satellite moved from the dayside to the nightside. In this case, the variation of the $X$ component seems to be large compared with the previous one. If the arc dynamics is coherent over the arc elongation, the ratio between the variation of $E x$ and that of $E y$ will give us an inclination angle $(\theta)$ with respect to noon-midnight direction, where a plus sign of $\theta$ indicates clockwise rotation, by using the following simple equation; i.e. 


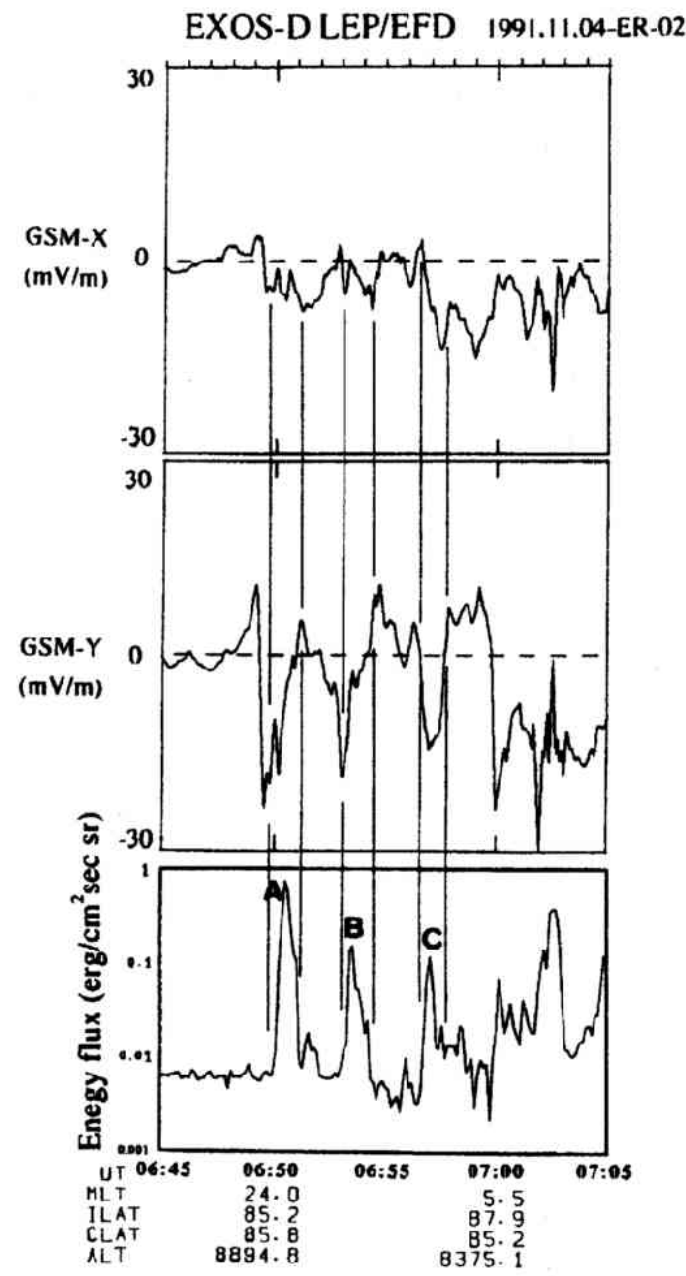

Fig. 5. Summary plot of the observations on Nov. 4, 1991.

$$
\tan \theta=\Delta E x / \Delta E y .
$$

Substituting $\Delta E x=-17 \mathrm{mV} / \mathrm{m}$ and $\Delta E y=-29 \mathrm{mV} / \mathrm{m}$, we obtain the angle; $\theta=30^{\circ}$. This means the arc is almost sun-aligned but slightly inclined clockwise with respect to the noon-midnight direction in this case. We have confirmed the consistency of the inclination from the all-sky images taken from Qaanaaq at that time.

\subsection{Response to IMF change}

Among 13 cases, only two cases are available for the comparison with IMF data. From Jan. 14 to Jan. 15,1990 , we observed a long lived SA. Figure 7 demonstrates the appearance of SA with respect to the history of the IMF. The SA persisted for more than 4 hours on that night. At 01:56UT, the SA disappeared due to the southward turning of the IMF at 00:55UT. There was a time delay of 1 hour in this case. At 01:33UT, the IMF turned northward and a SA appeared at 02:35UT again with a delay of about 1 hour. After the IMF turned southward at 02:03UT, the SA disappeared at 03:08UT.

Another day is Dec. 29, 1989. On that day SA disappeared from the field of view at 10:10UT due to 


\section{EXOS-D LEP/EFD}

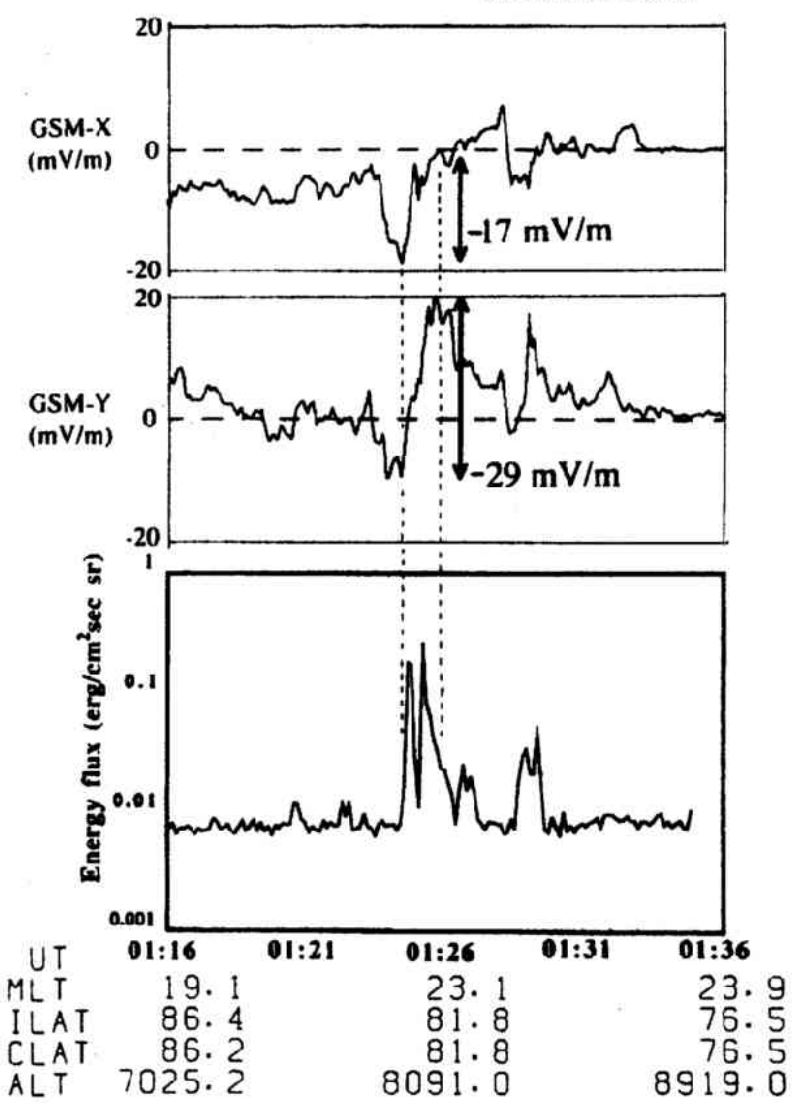

Fig. 6. Same format as in Fig. 5. Observation was made on Jan. 15, 1990.

14 Jan 1990

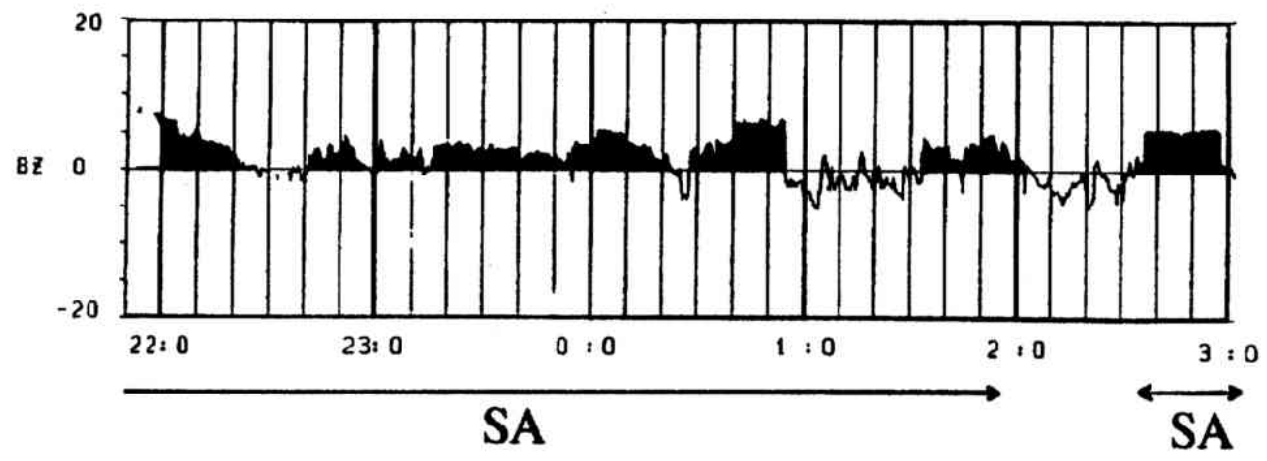

Fig. 7. History of IMF $B z$ on the night of Jan. 14-15. Intervals with $B z>0$ are marked in black. Akebono crossed this long lived SA at 01:25 UT. 
the southward turning of the IMF at 09:40UT with a delay of $30 \mathrm{~min}$. At 16:10UT, the IMF turned northward and a SA appeared at 17:00UT again with a time delay of $50 \mathrm{~min}$. SA decayed out of field of view at 22:00UT due to the southward turning of IMF at 19:35 with a delay of $25 \mathrm{~min}$.

\section{Relation of Patch Movement and Convection}

Patches of enhanced ionization, drifting across the polar cap region in the anti-sunward direction, have been observed from Qaanaaq station during geomagnetically active periods. Figure 8 is a sketch based on all-sky images at $630.0 \mathrm{~nm}$ and its projection to the CG coordinate. Patches are shown in black, showing the elongation oriented at around 45 degree with respect to the noon-midnight direction. They are clearly moving in the anti-sunward direction and also in the dawnward direction with a speed of 350 $\mathrm{m} / \mathrm{sec}$. The shape of patches changed a little bit as they moved. The moving velocity has been calculated from the movement of the center of patch.

At the same time, Akebono traversed the polar cap from the post noon (MLT $=14 \mathrm{~h}$ ) to the midnight $(\mathrm{MLT}=0 \mathrm{~h}$ ) having its closest approach to Qaanaaq at 00:03UT on Jan. 9, 1992. Top panel in Fig. 9 shows the $X$ (sunward) component of the electric field, and the middle one shows its $Y$ (duskward) component. The satellite entered the polar cap at 23:52UT, which was identified by the particle signature observed with the LEP instrument shown below. In the polar cap region, both the $X$ and $Y$ components of the electric fields had almost constant values, showing the very uniform convection at least along the satellite pass. Their values are $-13 \mathrm{mV} / \mathrm{m}$ and $17 \mathrm{mV} / \mathrm{m}$ for $X$ and $Y$, respectively, showing that the convection velocity is approximately $350 \mathrm{~m} / \mathrm{sec}$ at the $250 \mathrm{~km}$ height, directed anti-sunward and dawnward. This is consistent with the movement of patches, whose velocity is about $350 \mathrm{~m} / \mathrm{s}$ and is directed anti-sunward and dawnward, too.

On the night of Dec. 30, 1989, Akebono satellite traversed the polar cap from the post noon (MLT $=16 \mathrm{~h})$ to the post midnight $(\mathrm{MLT}=2 \mathrm{~h})$, having its closest approach to Qaanaaq at 22:45UT. The top panel in Fig. 10 shows the $X$ (Sunward) component of the electric field, and the bottom one shows the $Y$ (duskward) component. The Satellite entered the polar cap region at 22:40UT. The decreasing trend that is seen in the magnitude of the electric field is due to the increase of the satellite height. At $6,000 \mathrm{~km}$ (22:54UT), the electric field was $-3 \mathrm{mV} / \mathrm{m}$ and $12 \mathrm{mV} / \mathrm{m}$ for $X$ and $Y$, respectively, so that the convection velocity was approximately $300 \mathrm{~m} / \mathrm{sec}$ and directed almost anti-sunward at the $250 \mathrm{~km}$ height.

\subsection{8-9 Qaanaaq}

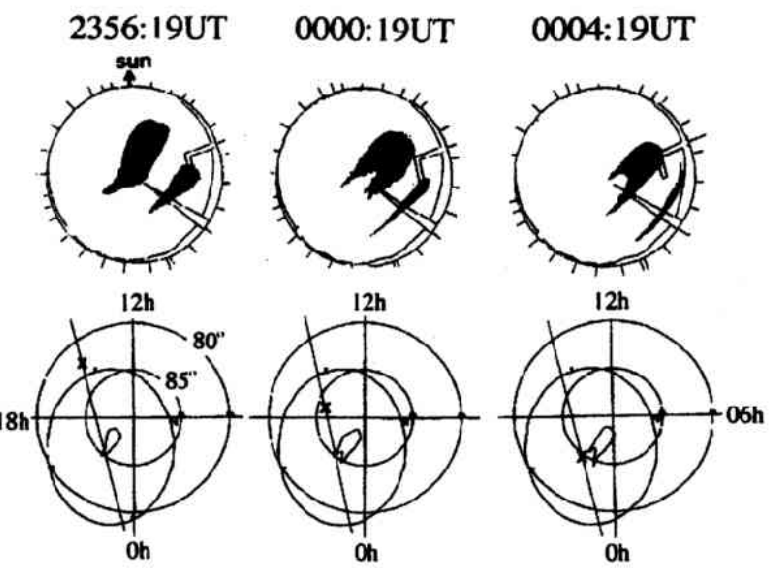

Fig. 8. Succeeding drawings based on all-sky images (top three) and their projection to the polar map. The satellite trajectory was drawn, in which the exact satellite location was marked " $x$ " for each time. 


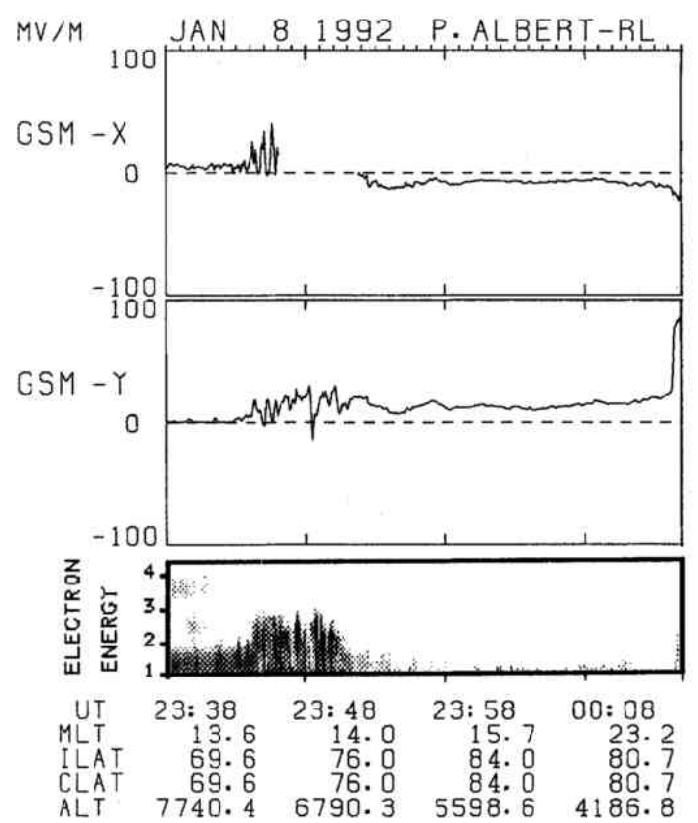

Fig. 9. Electric field data obtained from EFD instrument and E-t diagram for precipitating electron. Observation was made on Jan. 8, 1992.

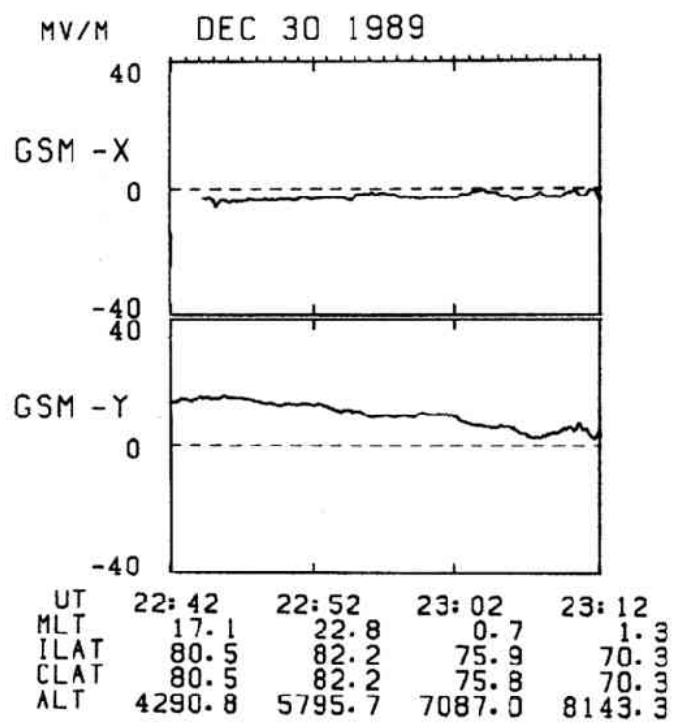

Fig. 10. Same format as in Fig. 9. Observation was made on Dec. 30, 1989.

On that night, we observed a total of 12 patches in a period from 22:00UT to 02:00UT from the Qaanaaq station. We have plotted the velocity obtained from the patch movement together with the digisonde drift velocity in Fig. 11. The velocity was in a range from $200 \mathrm{~m} / \mathrm{sec}$ to $530 \mathrm{~m} / \mathrm{sec} \mathrm{in} \mathrm{this} \mathrm{interval.}$ Akebono traversed the polar cap from 22:42UT to 23:12UT as mentioned previously. During that time, 


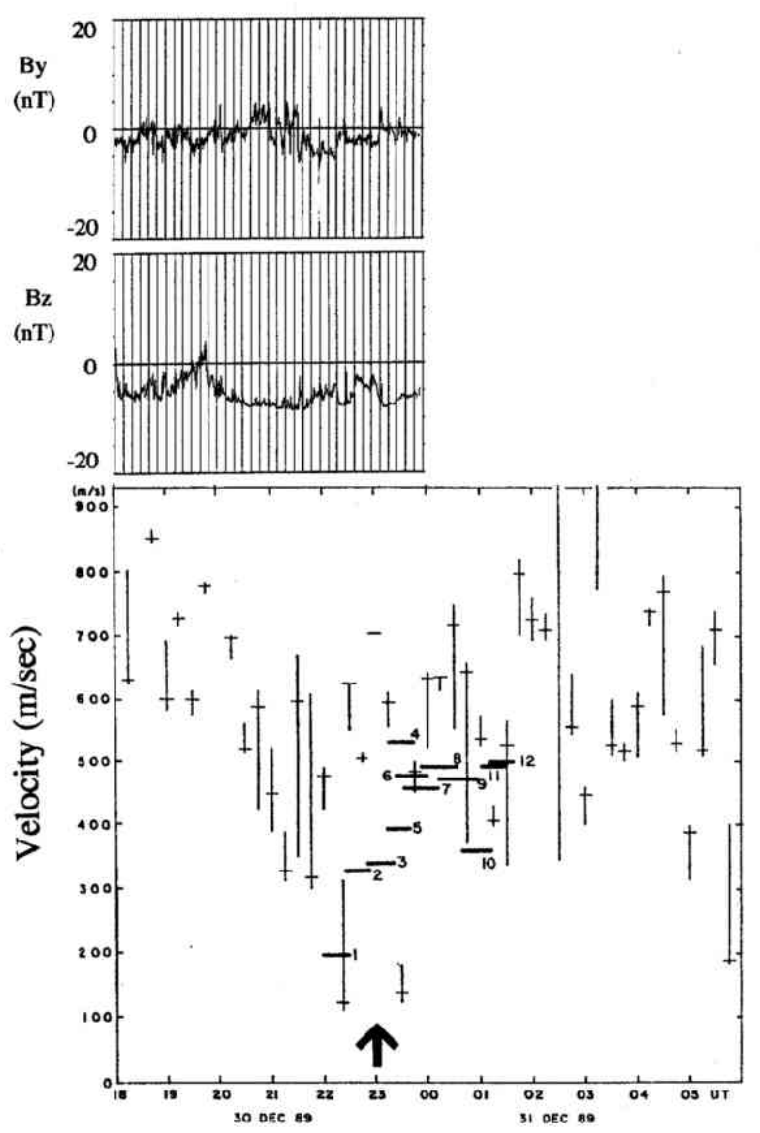

Fig. 11. Patch velocities (horizontal bars) calculated with the patch-tracing with digisonde results (indicated by plus signs) together with IMF $B y$ and $B z$.

patches No.2 and No.3 were observed in the Qaanaaq field of view, showing a velocity of about 320 $\mathrm{m} / \mathrm{sec}$. The direction of the patch movement was within $0^{\circ}$ to $-10^{\circ}$ from the anti-sunward direction (minus sign means counter clockwise rotation, see Fukui et al., 1994). Thus the movement of the patch agrees with the convection in this case, too. We have also looked at corresponding IMF By data (see the top of Fig. 11). From 21:35UT to 00:00UT, $B y$ was negative ( $-3 \mathrm{nT}$ on average). When $B y$ is negative, the polar cap convection takes a dawnward component. The results are consistent with the movement of patches as seen from Qaanaaq and the convection observed from the Akebono satellite.

There was a variety in the movement; although most of patches drifted anti-sunward, some ceased to drift within the ASC field of view. In 6 cases out of 19 cases, a patches did not demonstrate movement. On these occasions, Akebono observed a very small convection field (less than $2 \mathrm{mV} / \mathrm{m}$ ), suggesting that the plasma convection ceased, being consistent with the stagnant patch.

\section{Discussion}

\subsection{Sun-aligned arcs}

We have analyzed the simultaneous observation data from the ground based instruments at Qaanaaq and from the Akebono satellite. Results demonstrated the good correspondence between the localized 
electron precipitations observed from LEP instrument on board the Akebono satellite and the multiple SAs observed by the ASC at Qaanaaq. The electrodynamical signature of SA was investigated with the electric field data together with the particle data. Simple electrodynamical signature was found in the SA region; velocity shear with $\operatorname{div} E<0$ is associated with the electron precipitations. The shear is dominant in the noon-midnight direction rather than the dawn-dusk direction. This means that the structure is elongated in the noon-midnight direction; i.e. sun-aligned. In some cases the structure has an offset angle with respect to the noon-midnight direction. Such cases were mostly seen in the noon sector of the polar cap region rather than the nightside polar cap region (Obara et al., 1996). Detailed studies will be made of this phenomenon in future.

Velocity structure and electrodynamics have been clarified in the previous work (Carlson, 1990; Obara et al., 1993, 1996). They have investigated near-Earth processes influencing the SAs. A remaining problem is to find the relationship between SAs and the topology and driving processes in the magnetosphere. In order to understand the problem better, we have surveyed particle characteristics in the vicinity of the SAs. In Fig. 3 we have observed three SAs. Peak energy fluxes for three arcs are 0.8 ( $\mathrm{erg} / \mathrm{cm}^{2} \mathrm{sec} \mathrm{sr}$ ), 0.15 and 0.1 , respectively, while the energy flux was about 0.1 (erg/ $\mathrm{cm}^{2} \mathrm{sec} \mathrm{st}$.) in the oval region. The average energy was in a range from $200 \mathrm{eV}$ to $300 \mathrm{eV}$ in the vicinity of SAs, while the average energy in the oval region was much higher; i.e. $400 \mathrm{eV}$ to $500 \mathrm{eV}$. This difference is significant, and suggests that the source region of SAs should be different from the plasmasheet. Obara et al. (1996) investigated the average energy of the SAs based on the larger bodies of observations. Result demonstrates that the average energy was less than $200 \mathrm{eV}$, suggesting the magnetosheath/mantle origin (Obara et al., 1996).

The existence of theta aurora (Frank et al., 1986) which is very bright, having a luminosity more than a few $\mathrm{kR}$, is well known. Frank et al. (1986) inferred that the particles producing the theta aurora should originate in the plasmasheet or plasmasheet boundary layer. However it seems that the theta aurora is seen only a few percent of the time. More commonly, during northward IMF, multiple SAs are observed in the polar cap (Hardy, 1984; Carlson, 1990; Valladares et al., 1994; Obara et al., 1994) almost half of the time. It seems unlikely that bifurcation or expansion of the classical plasma sheet into polar latitudes as suggested by Frank et al. (1986) or Meng et al. (1981) for the theta configuration could occur so frequently during northward IMF condition, since it will take more energy to deform the shape of the plasmasheet. Consequently, we suggest the possibility of local plasma entry into the polar cap from the magnetosheath/ mantle during northward IMF conditions as the source of common sun-aligned arcs.

Of particular interest would be the response to IMF $B z$ changes, since this should provide valuable insights to the magnetospheric topology and coupling processes to the solar wind. According to our results, there is a systematic time delay in a range from $25 \mathrm{~min}$. to 1 hour. Such delay is consistent with the results of Rodriguez et al. (1995), who studied a winter of Qaanaaq ASC images and IMF data. The effect of $B z$ reversal, which creates or distracts SAs, involves the introduction of new flux tubes. Time lags may be determined (in part) by the convection velocity across the polar cap. An evidence that supports this scenario is that the SA decays from dayside (Rodriguez, private communication). The most SAs start to disappear from the dayside, but there are some exceptions which disappear from nightside. The disappearance of arcs is closely related to the decay of velocity shear, so more experimental works on the coherence of the dynamics over its length should be performed.

\subsection{Patches}

It has been reported that, during southward IMF, plasma islands having a size of a few hundred $\mathrm{km}$ and density enhancement of a factor 3-10 move rapidly across the polar cap region in a generally antisunward direction (Weber et al., 1984). Throughout the last decade, high latitude plasma has been observed by the digital ionosonde, and the relationship of convection velocity on the sign of $B z$ and $B y$ of IMF has been found (Buchau et al., 1988). Recently Fukui et al. (1994) compared the polar cap plasma motion from ASC images and plasma drift as measured by the digisonde, and confirmed their consistency. We have conducted a follow-up study by using the Akebono data and confirmed Fukui's result. 
It has been shown that the patches are transported into the polar cap region, rather than being formed locally in the polar cap by precipitation (Weber et al., 1984). We have confirmed this using the Akebono observations. As expected, the Akebono LEP instrument observed no significant electron precipitation above the patch regions.

The plasma with high density should be produced in the sunlit polar cusp, enter and traverse the polar cap region. The process which divides the plasma into islands remains unanswered. Recently Sojka et al. (1994) reported that patches are associated with changes of the IMF By polarity during the periods of southward IMF condition. As the IMF By variation changes, the plasma convection pattern redirects the plasma forming islands with high density. We actually observed many patches (12 patches) from Qaanaaq on the night of Dec. 30,1989, but the By polarity did not change very much as shown in the top of Fig. 11. Alternatively, sudden enhancement of convection velocity in the cusp region may introduce the plasma island with high density into the polar cap region. We will next study this possibility by using satellite and ASC data together with IMF data.

\section{Summary}

In Summary, the detailed processes of formation and movements of sun-aligned ares as well as patches were investigated based on the simultaneous observation data from the Akebono (EXOS-D) satellite and ground based instruments at Qaanaaq. Results demonstrated the electromagnetic structure of sun-aligned arcs and confirmed time delay of appearance and disappearance of sun-aligned arcs in response to IMF change. As for patches, there was a variety in terms of their movement; although most of patches drifted anti-sunward, some ceased to drift within the ASC field of view. Results of comparison between patch movement observed from the ground and the convection electric field observed from Akebono demonstrated a general consistency between them.

The Akebono satellite was launched by the Inst. of Space and Astronautical Science from Kagoshima Space Center (KSC) on February 22, 1989 and is still working. We thank Akebono tracking team very much for their extensive efforts. Authors wish to express their deep thanks to Prof. Nishida for advise and suggestions. J. V. Rodriguez was supported by a National Research Council-AFOSR Research Associateship. The work at Boston College was supported by Phillips laboratory contact F19628-90-0007 and by NSF grant ATM-9404088. We thank the Danish commission for Scientific Research in Greenland for permission to conduct experiments at Qaanaaq under continuing project A16-91.

\section{REFERENCES}

Buchau, J. D., W. Reinisch, E. J. Weber, and J. G. Moore, Structure and dynamics of the winter polar cap $F$ region, Radio Sci., 18, 995-1010, 1983.

Buchau, J. D., B. W. Reinisch, D. N. Anderson, E. J. Weber, and C. Dozois, Polar cap plasma convection measurements and their relevance to the real-time modeling of the high latitude ionosphere, Radio Sci., 23, 521-536, 1988.

Carlson, H. C., Dynamics of the quiet polar cap, J. Geomag. Geoelectr., 42, 697-710, 1990.

Frank, L. A., J. D. Craven, D. A. Gurnett, S. D. Shawhan, D. R. Weimer, J. L. Burch, J. D. Winningham, C. R. Chappell, J. H. Waite, R. A. Heelis, N. C. Maynard, M. Sugiura, W. K. Peterson, and E. G. Shelley, The theta aurora, J. Geophys. Res., 92, 3177-3224, 1986.

Fukui, K., J. Buchau, and C. E. Valladares, Convection of polar cap patches observed at Qaanaaq, Greenland during the winter of 1989-1990, Radio Sci., 29, 231-248, 1994.

Hardy, D. A., Intense fluxes of low-energy electrons at geomagnetic latitude above $85^{\circ}$, J. Geophys. Res., 89, 3883-3892, 1984.

Hayakawa, H., T. Okada, M. Ejiri, A. Kadokura, Y.-I. Kohno, K. Maezawa, S. Machida, A. Matsuoka, T. Mukai, N. Nakamura, A. Nishida, T. Obara, Y. Tanaka, F. S. Mozer, G. Hearendel, and K. Tsuruda, Electric field measurement on the Akebono (EXOS-D) satellite, J. Geomag. Geoelectr., 42, 371-384, 1990.

Meng, C.-I., The auroral electron precipitation during extremely quiet geomagnetic conditions, J. Geophys. Res., 86, 4607-4622, 1981.

Mukai, T., N. Kaya, E. Sagawa, M. Hirahara, W. Miyake, T. Obara, H. Miyaoka, S. Machida, H. Yamagishi, M. Ejiri, H. Matsumoto, and T. Itoh, Low energy charged particle observations in the "auroral" magnetosphere: First results from the 
Akebono (EXOS-D) satellite, J. Geomag. Geoelectr., 42, 479-496, 1990.

Obara, T., K. Syutou, T. Kato, T. Mukai, H. Hayakawa, K. Tsuruda, T. Abe, R. Fujii, K. Hashimoto, E. Kaneda, E. Sagawa, A. Morioka, and H. Oya, A new system for operation and data handling of Akebono (EXOS-D) satellite, J. Geomag. Geoelectr., 42, 565-577, 1990.

Obara, T., T. Mukai, H. Hayakawa, A. Nishida, K. Tsuruda, S. Machida, and H. Fukunishi, Akebono (EXOS-D) observations of small-scale electromagnetic signatures relating to polar cap precipitation, J. Geophys. Res., 98, 11153-11159, 1993.

Obara,T. , T. Mukai, H. Hayakawa, K. Tsuruda, A. Matsuoka, and A. Nishida, Relation of electric field and particle precipitation in the polar cap region, Antarctica Record, 38, 148-156, 1994 (in Japanese).

Obara, T., T. Mukai, H. Hayakawa, K. Tsuruda, A. Matsuoka, and A. Nishida, Signature of electric field associated with localized electron precipitations in the polar cap region-Akebono (EXOS-D) results, J. Geomag. Geoelectr., 48, 327-335, 1996.

Rodriguez, J., K. Fukui, and C. E. Valladares, Response of Sun-aligned Arcs to the IMF north-south polarity change, IAGA XXI general assembly, 1995.

Sojka, J. J., M. D. Bowline, and R. W. Schunk, Patches in the polar ionosphere: UT and seasonal dependence, J. Geophys. Res., 99, 14959-14970, 1994.

Valladares, C. E., H. C. Carlson, and K. Fukui, Interplanetary magnetic field dependency of stable sun-aligned polar cap arcs, J. Geophys. Res., 99, 6247-6272, 1994.

Weber, E. J., J. D. Buchau, J. G. Moore, J. R. Sharber, R. C. Livinston, J. D. Winningham, and B. W. Reinish, $F$ layer ionization patches in the polar cap, J. Geophys. Res., 89, 1683-1694, 1984. 